\title{
Les plateformes numériques aux Pays-Bas et la jurisprudence travailliste
}

\section{Hanneke Bennaars et Gerrard Boot}

\section{(2) OpenEdition}

1 Journals

\section{Édition électronique}

URL : https://journals.openedition.org/rdctss/1555

DOI : 10.4000/rdctss. 1555

ISSN : 2262-9815

Éditeur

Centre de droit comparé du travail et de la sécurité sociale

\section{Édition imprimée}

Date de publication : 1 juin 2019

Pagination : 60-67

ISSN : 2117-4350

\section{Référence électronique}

Hanneke Bennaars et Gerrard Boot, « Les plateformes numériques aux Pays-Bas et la jurisprudence travailliste », Revue de droit comparé du travail et de la sécurité sociale [En ligne], 2 | 2019, mis en ligne le 01 novembre 2021, consulté le 11 novembre 2021. URL : http://journals.openedition.org/rdctss/1555 ; DOI : https://doi.org/10.4000/rdctss. 1555

\section{cc)}

Revue de droit comparé du travail et de la sécurité sociale est mise à disposition selon les termes de la Licence Creative Commons Attribution - Pas d'Utilisation Commerciale - Pas de Modification 4.0 International. 


\section{LES PLATEFORMES NUMÉRIQUES AUX PAYS-BAS ET LA JURISPRUDENCE TRAVAILLISTE}
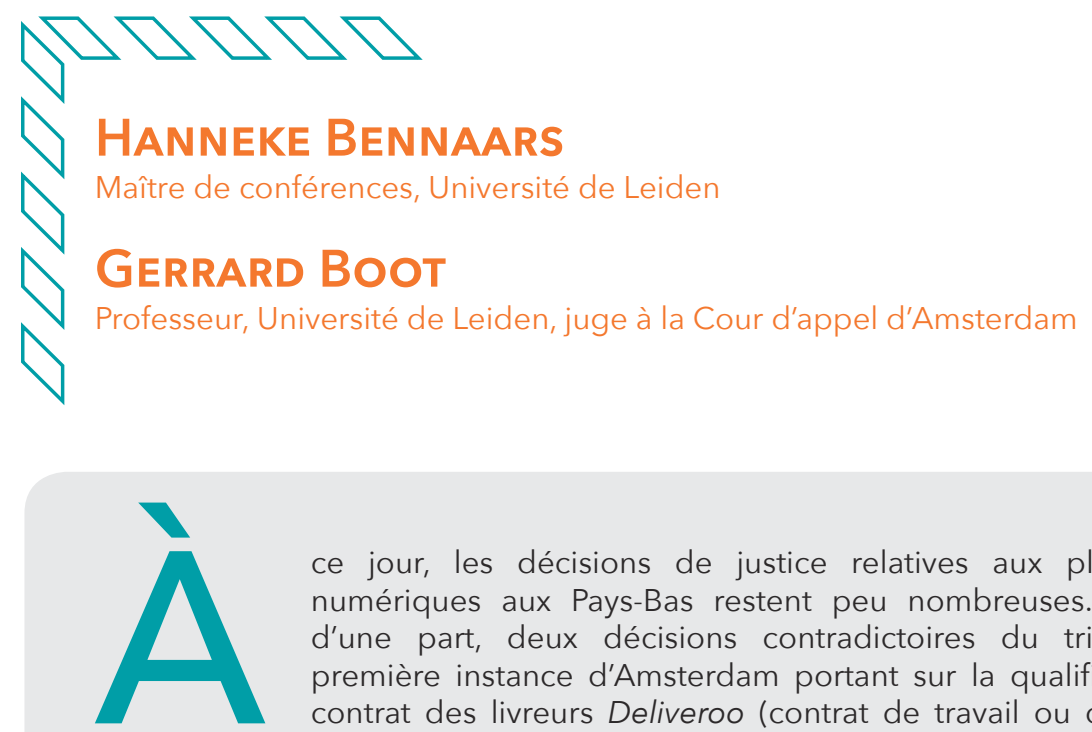

ce jour, les décisions de justice relatives aux plateformes numériques aux Pays-Bas restent peu nombreuses. II existe, d'une part, deux décisions contradictoires du tribunal de première instance d'Amsterdam portant sur la qualification du contrat des livreurs Deliveroo (contrat de travail ou contrat de prestation de services) et, d'autre part, deux décisions du tribunal de première instance d'Amsterdam et d'Utrecht concernant la classification des plateformes numériques de location touristique (Booking.com et Booker.BV). Dans ces deux dernières affaires, la question posée au tribunal ne concernait pas la qualification du contrat des travailleurs mais la question de savoir si les plateformes étaient ou non des agences de voyage. Une réponse positive entraîne de facto l'application du régime de retraite du secteur dont relèvent les agences de voyage et qui est obligatoire pour tous les employeurs du secteur. Les deux tribunaux ont rendu des jugements opposés. A deux reprises et sur les deux problématiques susmentionnées, il n'y a pas eu de consensus prétorien.

La question de la qualification d'une convention comme contrat de travail ou non, a des impacts et des conséquences qui divergent selon les cas. Cette question peut faire l'objet d'une procédure civile entre l'employeur et le salarié. Elle peut aussi être pertinente dans le cas où existe une convention collective avec un régime de retraite applicable aux salariés. Cette question peut également avoir des conséquences au regard du régime fiscal applicable. Si tel est le cas, l'employeur aux Pays-Bas doit retenir sur les salaires des impôts et des cotisations sociales. Enfin, la question de savoir si un contrat est bien un contrat de travail peut être posée dans le secteur public, par exemple pour établir si les horaires de travail correspondent à la réglementation en vigueur. 
Une procédure civile est initiée par des parties civiles. C'est le travailleur qui, souvent, prétend que son contrat est un contrat de travail. Aux Pays-Bas, il est également possible qu'un syndicat soit à l'origine de la procédure et agisse au nom de ses membres ou bien au nom des travailleurs en général (sur la base de l'article 3:305a CC).

Une procédure sur l'application d'une convention collective peut également être engagée par un salarié seul, ou bien par le syndicat. Souvent, la convention collective crée un organisme qui contrôle la bonne application de la convention. Cet organisme peut être à l'origine d'une procédure contre les employeurs. Si la convention collective prévoit un régime de retraite, les caisses de retraite peuvent aussi saisir le juge pour requalifier des contrats. Cela se produit assez souvent : la caisse de retraite réclame le versement de cotisations de retraite à l'entreprise car elle estime que les travailleurs sont embauchés sur la base d'un contrat de travail, alors que l'autre partie (le prétendu employeur) assure à l'inverse que le contrat est conclu avec des autoentrepreneurs ${ }^{1}$.

Lorsque la qualification de contrat de travail est retenue, l'employeur doit prélever des impôts sur les salaires, pour ensuite les reverser à l'administration fiscale. Lorsqu'il ne le fait pas, cette administration peut l'y contraindre au moyen d'une amende. L'employeur peut alors adresser une réclamation au percepteur. Si l'administration ne reconsidère pas la décision, l'employeur peut intenter une procédure devant le tribunal fiscal. En 2011, la Cour de cassation a aligné la jurisprudence de ce tribunal sur celle des juridictions civiles concernant la définition des contrats de travail.

Dès lors, il est possible de contester l'application de la loi sur la sécurité et la santé au travail ou celle sur la durée du travail. Dans ce cadre, il faut également établir le statut de travailleur : salarié ou non. Mais, même si le travailleur n'a pas le statut de salarié, ces lois peuvent néanmoins s'appliquer. Les procédures sur ce sujet sont plus rares que les conflits susmentionnés.

Les conséquences de la qualification d'un contrat diffèrent selon la nature du conflit ou de la procédure. Dans une procédure civile et dans l'hypothèse où le contrat serait qualifié de contrat de travail, le travailleur aura tous les droits y relatifs, comme le droit d'être rémunéré pendant une période de maladie ou de vacances, la protection contre le licenciement, mais aussi des indemnisations pour un accident de travail. Dans certaines conditions, les autoentrepreneurs peuvent également revendiquer ces indemnités. Cet article analyse les deux décisions : les jugements Deliveroo (I) et les jugements Booking et Booker (II).

\section{I - DELIVEROO : LA REQUALIFICATION PAR LE JUGE DU CONTRAT DES LIVREURS À VÉLO}

\section{A - L'Économie collaborative aux Pays-Bas}

Comme dans la plupart des pays européens, la qualification du contrat liant un opérateur (livreur, chauffeur, coursier) à une plateforme numérique est au centre du débat juridique. Aux Pays-Bas, la plupart des travailleurs des plateformes sont livreurs de repas (34\%), chauffeurs de taxi (14\%) ou fournissent des services à la personne (en particulier

1 Cour de Cassation Stipp; Cour de Cassation Rotterdamse Thuiszorg. 
pour l'entretien de la maison et les travaux ménagers, 14\%). Fin 2017, 0,4\% de la population active néerlandaise travaille dans l'économie des plateformes numériques (soit 34000 personnes) ${ }^{2}$. Les plateformes revêtent des modèles d'entreprise divers. Premièrement, la plateforme numérique peut fonctionner comme un "billard électronique ». Dans ce cas, elle ne fait qu'offrir un lien électronique pour des annonces, parfois combiné avec un moteur de recherche plus ou moins avancé. En général, ces sites web sont surtout utilisés par des travailleurs indépendants. Le rôle de la plateforme est alors limité ; elle ne se mêle pas de la relation entre le travailleur et le consommateur (la personne qui a besoin du service). Ce type de plateforme se retrouve principalement dans le domaine des menuisiers, plombiers, entrepreneurs de construction, et ciblent surtout les particuliers comme clients. Deuxièmement, il existe des plateformes de mise en relation, qui ont le fonctionnement d'une organisation intermédiaire et dont le rôle est plus important que les premières. Ces plateformes s'immiscent dans la relation contractuelle établie entre la personne qui réalise le travail et le client. C'est elle qui tarifie le service rendu, même si le travailleur et le client ont conclu un contrat (en général un contrat de prestations de services). L'implication de la plateforme peut se situer au niveau administratif ou pécuniaire (elle peut percevoir un pourcentage sur chaque service rendu). On retrouve cette catégorie de plateforme essentiellement dans le domaine des services à la personne (ménage, garde d'enfants, etc.). Troisièmement, il existe des plateformes qui organisent un service que des travailleurs rémunérés sont chargés de rendre. Les plateformes de livraison de repas, comme Deliveroo, se situent dans cette catégorie. Les modèles d'entreprises divergent. Certaines plateformes offrent aux livreurs un contrat de travail, alors que d'autres demandent aux livreurs d'être enregistrés comme autoentrepreneurs et souscrivent un contrat de prestations de services.

Deliveroo est entré sur le marché néerlandais en 2015 en proposant aux livreurs des contrats de travail (à durée déterminée). En 2017, Deliveroo a décidé de ne plus renouveler les contrats existants ni d'offrir de contrats de travail aux nouveaux livreurs, mais d'utiliser des contrats de prestations de services. A partir de février 2018, il n'y avait donc plus aucun livreur salarié travaillant pour Deliveroo. Depuis cette date, la Cour de première instance d'Amsterdam a statué à deux reprises sur la qualification du contrat. Les décisions ne sont pas concordantes : en juillet 2018, la Cour a estimé que le contrat entre le livreur et la plateforme devait être qualifié de contrat de prestations services. En janvier 2019, la même Cour est parvenue à une décision contraire : le contrat fut requalifié en contrat de travail.

\section{B - DELIVEROO I ${ }^{3}$ ET $I I^{4}$ : DES FAITS SIMILAIRES}

Dans les deux affaires, la Cour a du se prononcer sur la qualification du contrat et la plupart des faits étaient similaires.

La société Deliveroo avait recours à un site web et à une application permettant aux restaurateurs partenaires d'être mis en relation, aux clients de passer commande de repas et d'être livrés par des coursiers à vélo. Il était également possible pour les restaurants de

2 Bas ter Weel, Siemen van der Werff, Hanneke Bennaars, Robert Scholte, Julie Fijnje, Mies Westerveld \& Ton Mertens, Rise and growth of the gig economy in the Netherlands, Amsterdam, SEO Economisch Onderzoek 2018, SEO-rapport n²018-37.

3 Tribunal d'Amsterdam, 23 juillet 2018, ECLI:NL:RBAMS:2018:5183.

4 Tribunal d'Amsterdam, 15 janvier 2019, ECLI:NL:RBAMS:2019:198. 
participer au réseau via leurs propres livreurs, tout en conservant la possibilité offerte par Deliveroo de faire livrer le repas par un livreur Deliveroo. Les commandes de repas étaient attribuées par un algorithme nommé «Frank». Les livreurs utilisaient une application pour recevoir les commandes. Cette application contient un système SSB (" self-service booking tool ») qui est utilisé par le livreur pour s'inscrire sur un « shift » proposé par Deliveroo. Hormis «Frank » et «SSB », il existe un système de bonus qui récompense le livreur lorsqu'il traite davantage de commandes. Ces trois systèmes étaient déjà en œuvre quand les livreurs travaillaient comme salariés.

Les deux périodes peuvent être représentées de la manière suivante:

\begin{tabular}{|c|c|}
\hline Contrat de travail & Contrat de prestations de services \\
\hline $\begin{array}{l}\text { Contrat à durée déterminée flexible, entre } 1 \text { et } \\
160 \text { heures par mois. } \\
\text { Salarié rémunéré à I'heure, au niveau du SMIC } \\
\text { national. }\end{array}$ & $\begin{array}{l}\text { Rémunéré à la livraison, tarif variable. } \\
\text { Payé sur la base d'une facture. } \\
\text { S'inscrire n'est pas obligatoire. } \\
\text { Sans être inscrit, le livreur peut librement se } \\
\text { présenter sur l'application. } \\
\text { Une fois inscrit, le livreur peut choisir de ne pas } \\
\text { se présenter sur l'application, de se présenter } \\
\text { plus tard ou de quitter l'application plus tôt. } \\
\text { Le livreur peut se faire remplacer par quelqu'un } \\
\text { d'autre. Le remplaçant doit avoir le droit de } \\
\text { travailler aux Pays-Bas et doit décliner son } \\
\text { identité auprès de Deliveroo. }\end{array}$ \\
\hline Obligations & Obligations \\
\hline $\begin{array}{l}\text { S'inscrire au moins une fois par semaine pour } \\
\text { un shift de soir et une fois pour un shift de week- } \\
\text { end. } \\
\text { Sans être inscrit d'avance, le livreur ne peut se } \\
\text { présenter sur l'application qu'en cas de déficit } \\
\text { de livreurs. } \\
\text { L'inscription doit être renouvelée chaque } \\
\text { semaine avant une date limite. } \\
\text { Pendant un shift, le livreur est obligé d'accepter } \\
\text { une commande. } \\
\text { Les livreurs utilisent leur propre vélo et leur } \\
\text { téléphone portable personnel. } \\
\text { Les vêtements et le sac à dos sont fournis par } \\
\text { Deliveroo. } \\
\text { II est interdit de travailler pour d'autres } \\
\text { plateformes dans le même domaine. }\end{array}$ & $\begin{array}{l}\text { Le livreur est obligé de s'enregistrer comme } \\
\text { autoentrepreneur auprès de la Chambre de } \\
\text { commerce et auprès de l'administration fiscale. } \\
\text { Tous les équipements utilisés par le livreur } \\
\text { doivent être conformes aux normes de sécurité. } \\
\text { Le livreur peut utiliser son propre matériel, } \\
\text { même avec les logos de plateformes rivales. }\end{array}$ \\
\hline
\end{tabular}




\section{C - Deliveroo I ET II : DES FAITS DIVERgENTS}

La première décision concernait un livreur, M. Ferwerda, qui avait travaillé pour Deliveroo sur la base d'un contrat de travail jusqu'en novembre 2017. Comme mentionné précédemment, Deliveroo avait déclaré, en 2017, mettre un terme aux contrats de travail. Initialement, M. Ferwerda bénéficiait d'un contrat de travail à durée déterminée jusqu'au 3 février 2018. Un jour après avoir reçu l'annonce de Deliveroo, début novembre 2017, il avait demandé à transformer son contrat de travail en un contrat de prestations de services. Mi-novembre, ce contrat fut donc signé et, en janvier 2018, M. Ferwerda engageait une procédure civile, ayant au total travaillé à peu près 12 heures pour Deliveroo entre le 15 novembre et le jour de l'assignation. La deuxième procédure a été intentée par le syndicat FNV, I'un des plus grands syndicats néerlandais. Aucun livreur n'était partie à cette instance, ce qui a entraîné un débat sur la recevabilité de la demande. Une partie de la décision y est consacrée, mais nous ne l'aborderons pas ici.

\section{D - DeLIVEROO I ET II - QUeLLES diffÉRENCES ENTRE LES DEUX DÉCISIONS?}

L'article 7:610 du Code civil définit le contrat de travail de manière impérative, c'est-àdire que dans l'hypothèse où tous les éléments de la définition sont réunis, la relation entre les parties est un contrat de travail. II ne relève pas du libre arbitre des parties de décider de la qualification du contrat. Ainsi, «Le contrat de travail est le contrat par lequel une partie, le salarié, s'engage à travailler pendant un certain temps pour le compte de l'autre partie, l'employeur».

La définition comprend donc quatre éléments: (i) une activité de travail, (ii) contre un salaire, (iii) pendant un certain temps, et (iv) pour le compte de l'autre partie (subordination). On peut déduire de cette définition - en particulier de l'expression «s'engage à»-que le salarié est obligé de travailler (durant les heures convenues). Sans cette notion d'engagement, il est très difficile de qualifier un contrat de contrat de travail. Un élément tout aussi important concerne l'obligation faite, en principe, au salarié d'exécuter personnellement le travail. Lorsque l'employeur y est favorable, le salarié peut se faire remplacer, mais il n'est pas d'usage de se faire remplacer dans un contrat de travail.

Deux arrêts importants de la Cour de cassation ont formé le cadre dans lequel cette définition doit être appréciée. Tout d'abord, il ne faut pas seulement examiner le texte du contrat, mais davantage étudier la pratique de travail pour établir les droits et obligations des parties. En outre, il faut tenir compte de l'intention des parties, de toutes les circonstances factuelles, et de la position sociale des parties. C'est surtout « l'intention des parties » qui prête à confusion. Il s'agit de leur intention sur les droits et obligations mutuels, et non sur la qualification du contrat.

C'est sur cette distinction que repose la première différence entre les deux décisions. Si dans les deux jugements la Cour cite les règles, elle accorde en revanche, dans la première décision, une valeur plus importante à l'intention des parties sur la qualification du contrat. Le contrat contient un grand nombre de clauses stipulant ou soulignant que les parties ne souhaitent pas conclure un contrat de travail mais bien un contrat de prestation de services, lequel génère souvent des libertés pour le livreur. Dans la deuxième décision, le juge prend plus de distance par rapport au contrat écrit, peut-être parce qu'il s'agit d'un contrat standard d'adhésion rédigé par Deliveroo. La Cour considère que le contrat écrit n'apporte pas de renseignements précis sur l'intention du livreur. Au mieux, on peut en conclure que le livreur était prêt à travailler dans ces conditions, mais pas davantage. 
Dans ces deux décisions, la Cour compare la situation d'avant novembre avec celle d'après. Dans Deliveroo I, la Cour juge ainsi qu'il existe de grandes différences au niveau de la liberté du livreur de choisir ses heures en s'inscrivant ou non sur un shift, ou à l'inverse de choisir de ne pas travailler sans avoir besoin de se justifier. Par ailleurs, un très faible contrôle est exercé sur le travail, même après novembre, et le livreur peut travailler pour d'autres plateformes. Ces éléments conduisent le juge à conclure qu'il existe un véritable contrat de prestations de services.

Le deuxième jugement aboutit à une tout autre conclusion. Tout d'abord, il est établi que la liberté de travailler ou non existe surtout en théorie. Les systèmes électroniques ( "Frank » et «SSB ») et le système de bonus obligent en effet le livreur à travailler beaucoup, ne serait-ce que parce qu'une inscription fréquente sur des shifts octroie des avantages importants. Contrairement à la décision Deliveroo $I$, il est jugé que les instructions données au niveau de l'exécution du travail ne sont pas négligeables, notamment dans la mesure où le travail est standardisé et donc assez simple. Si l'enregistrement auprès de la Chambre de commerce constitue en général une indication forte de l'indépendance de la personne, la Cour précise néanmoins que, dans ce cas précis, on ne peut pas attacher une valeur trop importante à cet enregistrement car il est consécutif à une demande de Deliveroo, rendue obligatoire pour le livreur, et ne constitue donc pas un indice fiable de l'indépendance du livreur. Dans le deuxième jugement, le tribunal souligne la quasi-absence d'un esprit d'entreprise et, de ce fait, en conclut que les livreurs ne peuvent pas être considérés comme de vrais autoentrepreneurs.

Deliveroo a annoncé vouloir faire appel contre la deuxième décision. C'est donc la Cour d'appel d'Amsterdam qui doit se prononcer sur la qualification du contrat des livreurs Deliveroo. Comme dans la seconde procédure, il est intéressant de souligner que c'est le syndicat qui était demandeur à l'instance et qu'aucun livreur n'était partie au procès. Selon la communication de Deliveroo dans les médias, aucun livreur ne s'est, à ce jour, présenté pour réclamer les droits résultant de la requalification en contrat de travail.

\section{II - LES PROCÉDURES DE CLASSIFICATION DE SECTEUR : BOOKING.COM, BOOKER.BV}

Nous avons expliqué ci-avant que les caisses de retraite peuvent engager une procédure aux fins de requalifier des contrats. Ces caisses disposent d'organes de gestion paritaires, c'est-à-dire qu'elles sont composées d'un nombre égal de membres représentant les syndicats et le patronat. Cette retraite est une forme de retraite complémentaire ( " deuxième pilier »), qui s'ajoute à la pension de retraite versée par l'Etat, et concerne parfois les travailleurs d'une entreprise, parfois ceux d'un secteur industriel. Dans ce dernier cas, toutes les entreprises du secteur concerné sont obligées de cotiser. En règle générale, il est possible pour une entreprise d'obtenir une exonération, dès lors qu'elle dispose d'un régime de retraite au moins comparable au régime de retraite collectif. Les cotisations pour les retraites sont assez élevées : les primes s'élèvent à 10-20\% du salaire. Lorsqu'une entreprise n'est pas soumise à une convention collective de secteur, elle n'est pas obligée non plus d'adhérer au régime de retraite du secteur. Par conséquent, les entreprises qui tentent de réduire au minimum leurs coûts de personnel, évitent la plupart du temps d'être liées par une convention collective. 
Dans ce contexte, on relève deux procédures portant sur la question de savoir si la convention collective des agences de voyage était, ou non, applicable. La première procédure concernait la plateforme numérique Booking.com. En utilisant Booking.com, des particuliers peuvent réserver des chambres d'hôtel ou des appartements, quasiment partout dans le monde. Ces derniers règlent souvent leur chambre sur place, mais il est également possible que la somme soit payée via Booking.com. La question pour les juges hollandais consistait à se demander si Booking.com était une agence de voyages, ou simplement une entreprise numérique. C'est une question cruciale car, dans d'autres secteurs industriels, le travail traditionnel a été remplacé par de nouvelles technologies. Bien que Booking.com écrive sur son site en Belgique «Booking.com, le leader mondial dans la mise en relation des voyageurs avec le plus grand choix d'hébergements incroyables où séjourner, a annoncé aujourd'hui l'essai pilote de Booking.com pour les Agents de Voyage, la première version d'une nouvelle plateforme conçue spécialement pour répondre aux besoins des agents de voyage ", il n'en demeure pas moins qu'aux Pays-Bas, Booking.com affirme ne pas être une agence de voyages. Le tribunal de première instance d'Amsterdam ${ }^{5}$ a suivi cet argument, mais son homologue à Utrecht ${ }^{6}$ en a décidé différemment dans un litige concernant Booker.BV, une plateforme similaire à Booking.com mais présente dans beaucoup moins de pays.

Un des arguments invoqué par le juge d'Amsterdam consistait à souligner qu'un grand nombre des salariés de Booking.com étaient des experts en technologie et non des agents de voyage. Le tribunal a également considéré que le site Booking.com n'est qu'un intermédiaire électronique. Les dispositions du régime de retraite dans ce secteur, qui précisent le degré d'influence de la convention, impliquent dans la définition d'agence de voyage que l'entreprise ait un rôle actif, allant bien au-delà de la fonction d'intermédiaire électronique. Selon le tribunal, ce rôle d'intermédiaire entraîne un rôle plus actif que celui prétendument joué par Booking.com.

Pour le tribunal à Utrecht, il était important de noter que le travail de Booker.BV consiste à offrir un logement touristique, et que cela correspond avec l'objectif d'une agence de voyages classique. Par conséquence, Booker pouvait recevoir une telle qualification, à l'inverse de ce qui avait été retenu pour son homologue Booking. Dans le cas de Booking.com, un appel a été interjeté auprès de la Cour d'appel d'Amsterdam. La décision est attendue dans le courant de l'été 2019.

5 Tribunal d'Amsterdam 30 décembre 2016, ECLI:NL:RBAMS:2016:9040.

6 Tribunal d'Utrecht 9 mai 2018, ECLI:NL:RBMNE:2018:2044. 


\section{PAYS-BAS}

En conclusion, bien que les décisions de justice sur le travail via des plateformes aux Pays-Bas soient limitées, en nombre et en portée, étant donné qu'il ne s'agit que de jugements de première instance, quelques conclusions provisoires peuvent néanmoins d'ores et déjà être tirées. Tout d'abord, on ne constate pas de tendance à traiter le cas des plateformes d'une manière spéciale. Les tribunaux utilisent les cadres juridiques connus même si, dans toutes les décisions, on insiste sur le fait qu'il s'agit de modèles d'entreprises nouveaux. Parfois, le tribunal renvoie au législateur la responsabilité d'agir. En outre, il convient de souligner que les tribunaux montrent une légère tendance à vouloir intégrer les aspects technologiques dans les cadres juridiques traditionnels. Motiver les livreurs à travailler, plutôt que leur donner directement des ordres, peut également constituer une subordination juridique ou une obligation de fournir la prestation de travail. 